\title{
RXTE confirmation of the intermediate polar status of IGR J15094-6649
}

\author{
O. W. Butters ${ }^{1,2}$, A. J. Norton ${ }^{2}$, K. Mukai ${ }^{3,4}$, and E. J. Barlow ${ }^{2}$ \\ 1 Department of Physics and Astronomy, University of Leicester, Leicester LE1 7RH, UK \\ e-mail: oliver.butters@star.le.ac.uk \\ 2 Department of Physics and Astronomy, The Open University, Walton Hall, Milton Keynes MK7 6AA, UK \\ 3 CRESST and X-ray Astrophysics Laboratory NASA/GSFC, Greenbelt, MD 20771, USA \\ 4 Department of Physics, University of Maryland, Baltimore County, 1000 Hilltop Circle, Baltimore, MD 21250, USA
}

Received 26 January 2009 / Accepted 13 March 2009

\section{ABSTRACT}

\begin{abstract}
Aims. We aim to establish the X-ray properties of the intermediate polar candidate IGR J15094-6649 and therefore confirm its inclusion into the class.

Methods. $42856 \mathrm{~s}$ of X-ray data from RXTE was analysed. Frequency analysis was used to constrain temporal variations and spectral analysis used to characterise the emission and absorption properties.

Results. A spin period of $809.7 \pm 0.6 \mathrm{~s}$ is present, revealed as a complex pulse profile whose modulation depth decreases with increasing X-ray energy. The spectrum is well fitted by either a $17 \pm 4 \mathrm{keV}$ Bremsstrahlung or $\Gamma=1.8 \pm 0.1$ power law, with an iron emission line feature and significant absorption in each case.

Conclusions. IGR J15094-6649 is confirmed to be an intermediate polar.
\end{abstract}

Key words. stars: binaries: general - stars: novae, cataclysmic variables - stars: individual: IGR J15094-6649 - X-rays: binaries

\section{Introduction}

Intermediate polars (IPs) are members of the cataclysmic variables class. They are thought to harbour a magnetic field strong enough to greatly influence the accretion from the main sequence star to the white dwarf, but not strong enough to synchronise the system. For this situation to occur the magnetic field is believed to be in the range of a few MG to tens of MG at the white dwarf surface. This has the effect of channelling the accreting material onto the magnetic poles of the white dwarf and causes a hot dense accretion column to form, which emits high energy X-rays. As the white dwarf spins, the absorbing column density in the line of sight varies; this gives rise to one of the defining characteristics of IPs - X-ray modulation at the spin period with a modulation depth which decreases as the X-ray energy increases. For an exhaustive review of CVs see e.g. Warner (1995).

The exact number of IPs is heavily dependent on the selection criteria used, but the lower end estimate is currently taken to be thirty three ${ }^{1}$.

In recent years hard X-ray telescopes (INTEGRAL and Swift) have unexpectedly found many known IPs and discovered several candidate systems. This has raised the question of whether a sample of hard X-ray selected candidate IPs would be different from the current soft X-ray selected population. With this in mind the candidate IGR J15094-6649 (hereafter J1509) is studied. This forms part of an ongoing survey to classify hard X-ray selected IPs (Butters et al. 2007, 2008).

\footnotetext{
${ }^{1}$ http://asd.gsfc.nasa.gov/Koji.Mukai/iphome/iphome. html (IP catalogue version 2008b).
}

\section{Previous observations of $\mathrm{J} 1509$}

$\mathrm{J} 1509$ was discovered in the INTEGRAL/IBIS survey as an unclassified object in the $17-60 \mathrm{keV}$ energy band (Revnivtsev et al. 2006).

Masetti et al. (2006) classified J1509 as an IP based upon optical spectra taken at the $1.5 \mathrm{~m}$ Cerro Tololo Interamerican Observatory (CTIO) in Chile.

Barlow et al. (2006) also reported INTEGRAL data, but in the 20-100 keV energy range this time. Both a Bremsstrahlung and a power law were fitted to the data in order to determine an identification. In both cases a good fit was found; Bremsstrahlung with $k T=13.8 \pm 5.1 \mathrm{keV}$ and power law with $\Gamma=3.6 \pm 0.8$. The flux was given as $1.38 \times 10^{-11} \mathrm{erg} \mathrm{s}^{-1} \mathrm{~cm}^{-2}$ in the $20-100 \mathrm{keV}$ band.

Very recently Pretorius (2009) published optical photometry and spectroscopy of J1509, along with four other candidate IPs from the INTEGRAL sample. She detected a clear radial velocity signal at a period of $5.89 \pm 0.01 \mathrm{~h}$ which was identified as the orbital period of the system, as well as a photometric modulation at $809.42 \pm 0.02 \mathrm{~s}$ which was taken to be the spin period of the magnetic white dwarf. These results provided very strong indications that J1509 is an IP, and detection of a commensurate pulse period in X-ray data would absolutely confirm its classification.

\section{Observations and data reduction}

Data were obtained from the RXTE satellite (Bradt et al. 1993) with the PCA instrument over two consecutive days, from 30th-31st December 2008. The total time on target was $42856 \mathrm{~s}$. Initial data reduction was done with the standard FTOOLS. Only the top layer of PCU2 was included in the measurements and we 


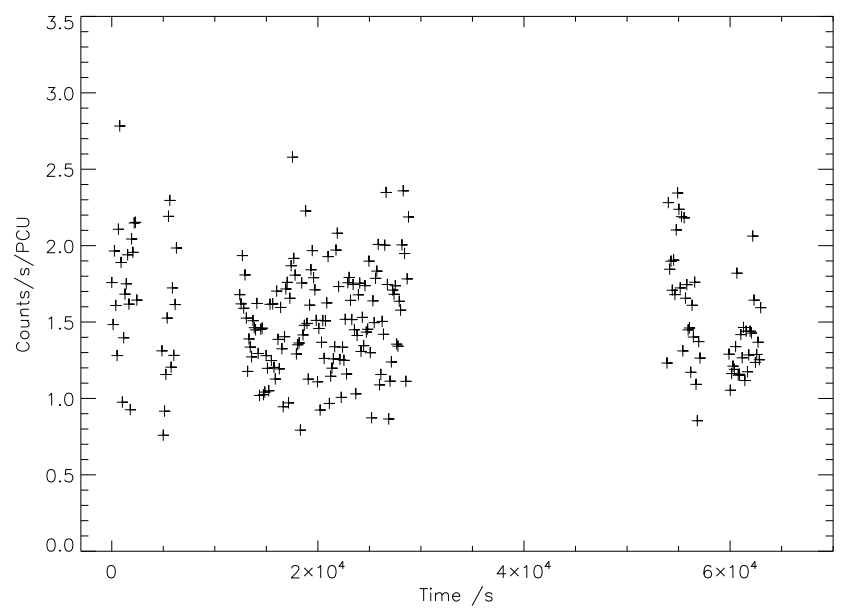

Fig. 1. 2-10 keV background subtracted light curve of J1509. The zero time corresponds to the start of the observations at JD 2454830.77312937984 . The data is binned into bins of $128 \mathrm{~s}$ width. The typical error on each point is 0.26 counts $\mathrm{s}^{-1} \mathrm{PCU}^{-1}$.

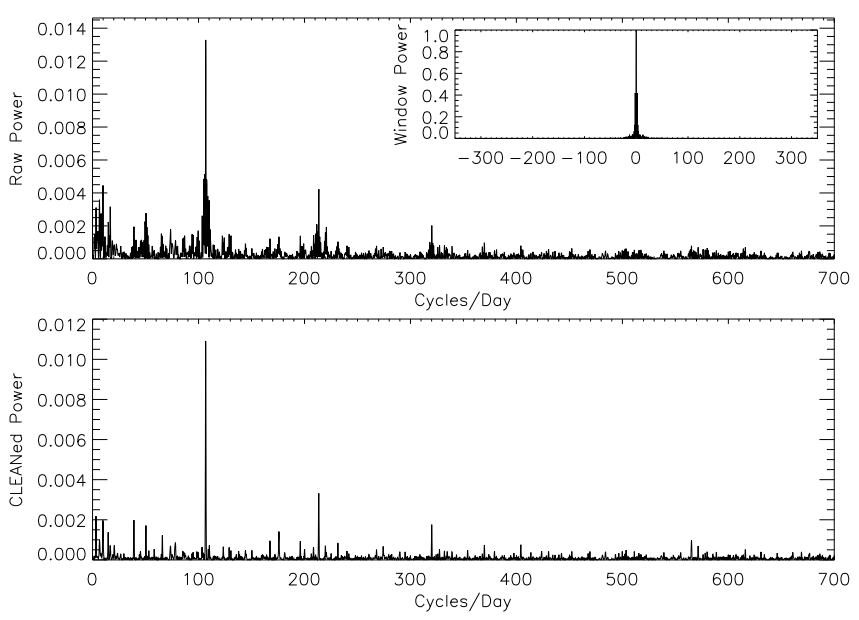

Fig. 2. 2-10 keV CLEANed periodogram. The upper plot shows the raw periodogram, with the window function inset; the lower plot shows the deconvolved (CLEANed) periodogram.

used the standard 2 mode data with a time resolution of $16 \mathrm{~s}$. Background subtracted light curves were constructed in four energy bands: $2-4 \mathrm{keV}, 4-6 \mathrm{keV}, 6-10 \mathrm{keV}$ and $10-20 \mathrm{keV}$, as well as a combined $2-10 \mathrm{keV}$ band for maximum signal-tonoise. A mean X-ray spectrum was also extracted.

\subsection{Light curve analysis}

In the $2-10 \mathrm{keV}$ energy band the raw count rate varied between 2.5 and 6.9 count $\mathrm{s}^{-1} \mathrm{PCU}^{-1}$. The background count rate, generated using the faint source background model, varied between 2.9 and 4.1 count $\mathrm{s}^{-1} \mathrm{PCU}^{-1}$. The background subtracted 2-10 keV light curve is shown in Fig. 1. The data were subsequently analysed with a variable gain implementation of the CLEAN algorithm (Lehto 1997) to discover any periodicities and discount any aliasing effects. The results of this are shown in Fig. 2.

A strong peak is evident in the CLEANed periodogram at approximately 107 cycles day $^{-1}$, in the $2-10 \mathrm{keV}$ energy band. Also present are its first and second harmonics at $\sim 214$ and $\sim 321$ cycles day $^{-1}$ respectively. Analysis of the second harmonic peak yields a fundamental pulsation period of $809.7 \pm 0.6 \mathrm{~s}$

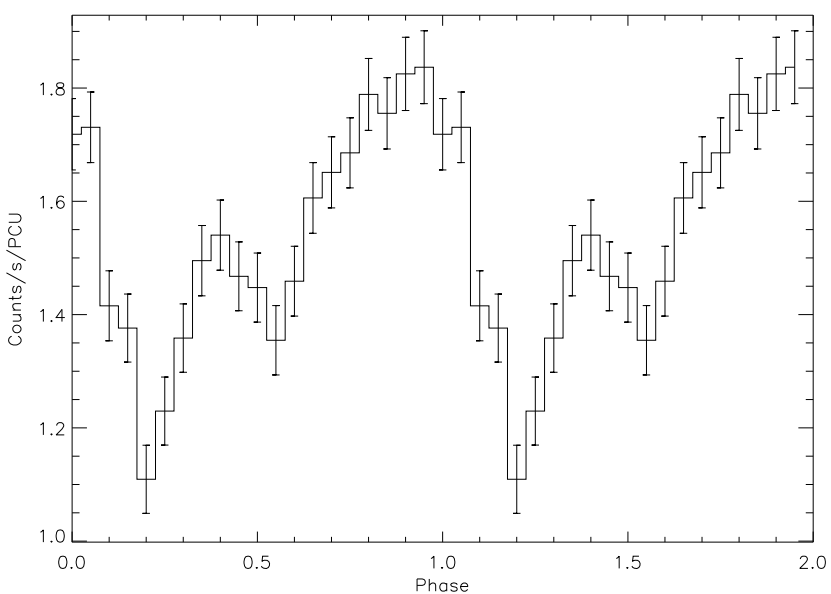

Fig. 3. 2-10 keV light curve folded at the $809.7 \mathrm{~s}$ period with an arbitrary zero point. Two cycles are shown for clarity.

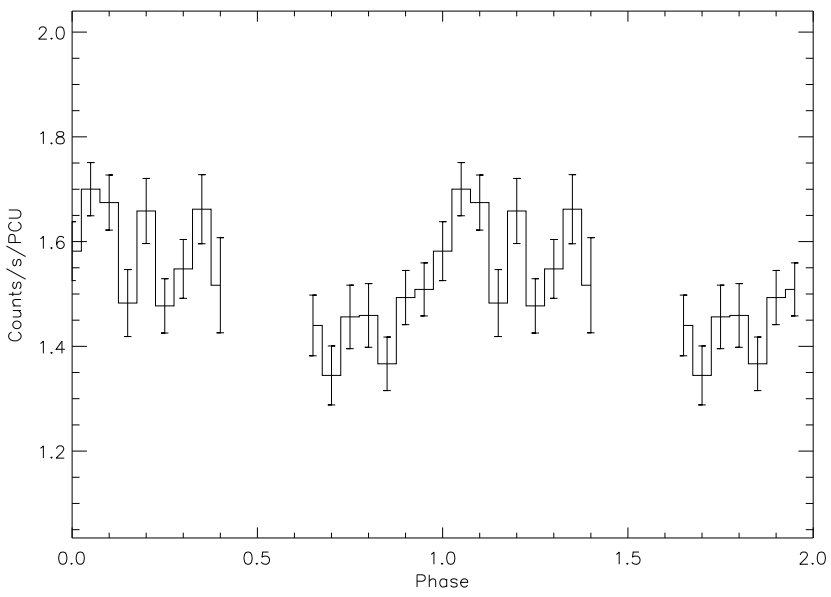

Fig. 4. 2-10 keV light curve folded at the $21204 \mathrm{~s}$ period of Pretorius (2009) with an arbitrary zero point. Two cycles are shown for clarity.

Table 1. Modulation depths of the pulse profile in different energy bands.

\begin{tabular}{ccc}
\hline \hline $\begin{array}{c}\text { Energy band } \\
(\mathrm{keV})\end{array}$ & $\begin{array}{c}\text { Modulation depth } \\
(\%)\end{array}$ & $\begin{array}{c}\text { Fitted mean } \\
\left(\mathrm{ct} \mathrm{s}^{-1} \mathrm{PCU}^{-1}\right)\end{array}$ \\
\hline $2-10$ & $15 \pm 1$ & 1.54 \\
$2-4$ & $27 \pm 3$ & 0.33 \\
$4-6$ & $16 \pm 2$ & 0.54 \\
$6-10$ & $9 \pm 2$ & 0.67 \\
$10-20$ & $7 \pm 4$ & 0.34 \\
\hline
\end{tabular}

(based on a Gaussian fit to the periodogram). This is in excellent agreement with the optical photometric period detected by Pretorius (2009). Each of the energy resolved light curves were folded at the $809.7 \mathrm{~s}$ period, and Fig. 3 shows the result in the $2-10 \mathrm{keV}$ energy band. The modulation depths of the pulse profile were then estimated by fitting a sinusoid to the folded data in each energy band and dividing the semi-amplitude by the fitted mean. The results of this are shown in Table 1.

There is no indication in the power spectrum of the spectroscopic orbital period previously reported by Pretorius (2009). Folding the X-ray light curve at the proposed orbital period yields a profile with no significant coherent modulation (see Fig. 4). 
Table 2. Bremsstrahlung (top) and power law (bottom) spectral fitting parameters of J1509. $n_{\mathrm{H}}$ (Galactic) $=0.2 \times 10^{22} \mathrm{~cm}^{-2}$.

\begin{tabular}{cccccccc}
\hline \hline $\begin{array}{c}n_{\mathrm{H}} \\
10^{22} \mathrm{~cm}^{-2}\end{array}$ & $\begin{array}{c}k T \\
\mathrm{keV}\end{array}$ & $\Gamma$ & $\begin{array}{c}\mathrm{Fe} \\
\mathrm{keV}\end{array}$ & $\begin{array}{c}\sigma_{\mathrm{Fe}} \\
\mathrm{keV}\end{array}$ & $\begin{array}{c}E W \\
\mathrm{keV}\end{array}$ & $\chi_{\text {reduced }}^{2}$ & $\begin{array}{c}\text { Flux }(2-10 \mathrm{keV}) \\
10^{-11} \mathrm{erg} \mathrm{cm}^{-2} \mathrm{~s}^{-1}\end{array}$ \\
\hline $1.6 \pm 1.0$ & $17 \pm 4$ & - & $6.4 \pm 0.1$ & $0.4 \pm 0.1$ & 0.8 & 0.7 & 1.5 \\
$3.0 \pm 1.3$ & - & $1.8 \pm 0.1$ & $6.4 \pm 0.1$ & $0.4 \pm 0.1$ & 0.9 & 0.8 & 1.5 \\
\hline
\end{tabular}
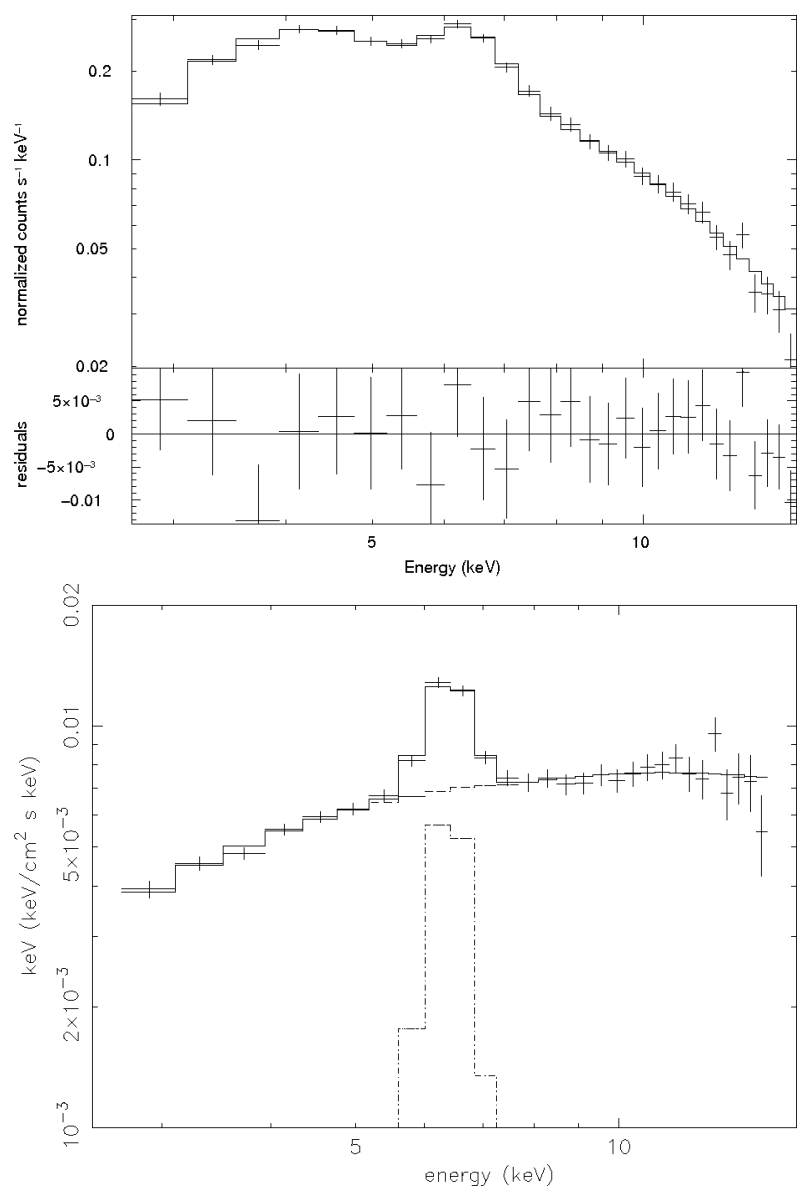

Fig. 5. Folded (top) and unfolded (in $v F_{v}$ representation, bottom) 2.5-15 keV mean spectrum fitted with a photoelectrically absorbed Bremsstrahlung plus iron line profile.

\subsection{Spectral analysis}

Analysis of the X-ray spectrum was carried out with the XSPEC package. Two models were used for fitting; a photoelectrically absorbed Bremsstrahlung, and a photoelectrically absorbed power law. Both models had an excess at approximately $6.4 \mathrm{keV}$, so a Gaussian was added to account for the iron line emission. Both models gave a good fit to the data (see Fig. 5 and Table 2).

\section{Discussion}

The X-ray period found here (809.7 s) is in agreement with the photometric pulsation period found by Pretorius (2009) and is typical of a white dwarf spin period in an IP. Furthermore, the increasing modulation depth with decreasing energy in the folded pulse profiles is an indication that an accretion column absorbing structure is present (Norton \& Watson 1989). The complex pulse profile and presence of strong harmonics in the power spectrum are reminiscent of the canonical IP FO Aqr (Beardmore et al. 1998), although unlike that system, there is here no evidence for an additional X-ray modulation at the beat period (841.5 s), which would be indicative of a stream-fed component to the accretion. We note that whilst some IPs exhibit the white dwarf spin period in their X-ray flux, they may show the beat period in optical photometry (for example, AO Psc). This arises due to reprocessing of the X-ray signal, probably from the face of the donor star. In the case of J1509 we can be confident that we are seeing the spin period of the white dwarf in both the optical and X-ray light curves. The length of the X-ray data set probably precludes the detection of the orbital period, or may indicate that the system is seen at relatively low inclination angle (Parker et al. 2005), so no such modulation is present.

Both spectral fits are good and the Bremsstrahlung model in particular is in agreement with that seen in the INTEGRAL data at higher energies (Barlow et al. 2006). The fit parameters are typical of those seen in other IPs. The column density is greater than the Galactic column density (as given by the HEASARC $n_{\mathrm{H}}$ estimator $^{1}$ ). This too is typical of IPs and is likely due to absorption by material within the accretion flow. The ROSAT Bright Source Catalogue has one other source in the RXTE field of view. A count rate for it was estimated using the webPIMMS ${ }^{2}$ tool and scaled according to the response of the detector. The count rate of this additional source was small $\left(\sim 0.04\right.$ counts $\left.\mathrm{s}^{-1} \mathrm{PCU}^{-1}\right)$ and therefore does not affect our result.

\section{Conclusion}

IGR J15094-6649 is confirmed as an IP and adds to the growing list of hard X-ray selected magnetic CVs discovered by INTEGRAL.

\section{References}

Barlow, E. J., Knigge, C., Bird, A. J., et al. 2006, MNRAS, 372, 224

Beardmore, A. P., Mukai, K., Norton, A. J., Osborne, J. P., \& Hellier, C. 1998, MNRAS, 297, 337

Bradt, H. V., Rothschild, R. E., \& Swank, J. H. 1993, A\&AS, 97, 355

Butters, O. W., Barlow, E. J., Norton, A. J., \& Mukai, K. 2007, A\&A, 475, L29

Butters, O. W., Norton, A. J., Hakala, P., Mukai, K., \& Barlow, E. J. 2008, A\&A, 487,271

Lehto, H. J. 1997, in Applications of time series analysis in astronomy and meteorology, ed. T. Subba Rao, M. B. Priestley, \& O. Lessi (London: Chapman and Hall)

Masetti, N., Morelli, L., Palazzi, E., et al. 2006, A\&A, 459, 21

Norton, A. J., \& Watson, M. G. 1989, MNRAS, 237, 853

Parker, T. L., Norton, A. J., \& Mukai, K. 2005, A\&A, 439, 213

Pretorius, M. L. 2009, MNRAS, in press [arXiv: 0901.2841]

Revnivtsev, M. G., Sazonov, S. Y., Molkov, S. V., et al. 2006, Astron. Lett., 32, 145

Warner, B. 1995, Cataclysmic variable stars, Cambridge Astrophys. Ser. (Cambridge, New York: Cambridge University Press)

\footnotetext{
1 http://heasarc.nasa.gov/cgi-bin/Tools/w3nh/w3nh.pl

2 http://www. ledas.ac.uk/pimms/w3p/w3pimms.html
} 